ApJL, in press

\title{
On the Formation of Compact Stellar Disks Around Sgr A*
}

\author{
Mark Wardle \\ Department of Physics, Macquarie University, Sydney NSW 2109, Australia \\ wardle@physics.mq.edu.au \\ Farhad Yusef-Zadeh \\ Department of Physics \&3 Astronomy, Northwestern University, Evanston, IL 60208, USA \\ zadeh@northwestern.edu
}

\begin{abstract}
The recent identification of one or two sub-parsec disks of young, massive stars orbiting the $\sim 4 \times 10^{6} \mathrm{M}_{\odot}$ black hole Sgr $\mathrm{A}^{*}$ has prompted an "in-situ" scenario for star formation in disks of gas formed from a cloud captured from the Galactic center environment. To date there has been no explanation given for the low angular momentum of the disks relative to clouds passing close to the center. Here we show that the partial accretion of extended Galactic center clouds, such as the $50 \mathrm{~km} \mathrm{~s}^{-1}$ giant molecular cloud, that temporarily engulf Sgr A* during their passage through the central region of the Galaxy provide a natural explanation for the angular momentum and surface density of the the observed stellar disks. The captured cloud material is gravitationally unstable and forms stars as it circularizes, potentially explaining the large eccentricity and range of inclinations of the observed stellar orbits. The application of this idea to the formation of the circumnuclear ring is also discussed.
\end{abstract}

Subject headings: accretion, accretion disks — Galaxy: center — ISM: clouds stars: formation

\section{Introduction}

A high concentration of mass, almost certainly a $\sim 4 \times 10^{6} \mathrm{M}_{\odot}$ black hole, is located at the very center of an evolved, centrally-concentrated stellar population and coincident with 
the bright compact radio source Sgr A* (e.g. Genzel et al. 2003; Schödel et al. 2003; Ghez et al. 2003; Reid \& Brunthaler 2004). The discovery of a young cluster of massive stars (Krabbe et al. 1991, 1995) in the hostile tidal environment within a parsec of Sgr A* is surprising (e.g. Morris 1993); even more remarkable is the discovery that the cluster consists of one or possibly two counter-rotating, thick stellar disks with surface density profiles scaling as the inverse square of the true distance from Sgr A* (Genzel et al. 2003; Paumard et al. 2006), although the existence of the second disk awaits independent confirmation (Lu et al. 2006). The inner and outer radii of the better-defined clockwise disk are $\approx 0.03$ and $0.3 \mathrm{pc}$, and the stellar ages are estimated to be $\sim 6 \mathrm{Myr}$, with the total mass of stars amounting to $\sim 1.5 \times 10^{4} \mathrm{M}_{\odot}($ Paumard et al. 2006).

There are two mechanisms favoured for the formation of a compact stellar disk around Sgr $A^{*}$. In one, a cluster of massive stars spirals into the central region because of tidal friction with the evolved stellar population centered on Sgr A*, and is tidally disrupted to form a stellar disk (Gerhard 2001; McMillan \& Portegies Zwart 2003; Portegies Zwart, McMillan \& Gerhard 2003; Kim et al. 2004; Gürkan \& Rasio 2005). The time scale for tidal friction exceeds the stellar ages unless the cluster is extraordinarily massive and compact (see Paumard et al. 2006), although recent work finds a faster inspiral so that this possibility is still open (Fujii et al. 2007). Nevertheless, this mechanism tends to produce a far more disordered stellar orbits than observed, as well as a population of massive stars shed from the cluster that should extend beyond $0.3 \mathrm{pc}$ from Sgr A*. In the second, "in-situ formation" scenario, an interstellar cloud is tidally disrupted and captured by Sgr A*, settles into a gravitationally unstable disk, and forms the stars that we see today (e.g. Levin \& Bolobordov 2003; Nayakshin \& Cuadra 2005). In-situ star formation overcomes the time scale issue and more naturally produces the gross kinematics of the observed disks. However, star formation within a kinematically cold disk produces stellar orbits that are less eccentric and more coplanar than observed, even accounting for gravitational scattering of newly-formed stars by other members of the new stellar population (Cuadra, Armitage \& Alexander 2008).

Simulations of this scenario generally start with the evolution of an initially gravitationally unstable disk or of a compact cloud in a close orbit around Sgr A*, implicitly assuming that formation of the stellar disk is initiated by the chance capture of an isolated, compact, low-angular momentum gas cloud. This, however, is unlikely to be so because of the compactness of the stellar disk relative to the scale of molecular clouds and cores. While the kinetic energy of a compact incoming cloud can be readily dissipated by shocks and subsequently radiated away, the net angular momentum of the cloud material remains unchanged. Thus the initial angular momentum must be very low indeed if the cloud is to circularize into a disk of radius $\lesssim 0.3$ pc. Instead, as recently noted by Yusef-Zadeh \& Wardle (2008), it is much more likely that the precursor disks are formed by the partial capture of an extended 
molecular cloud that temporarily engulfs Sgr A* on a passage through the Galactic center rather than passing to one side of it. Simulations by Mapelli et al. (2008) of the capture of a cloud on an almost radial orbit suggests that star formation during such an event will occur before the disk has fully circularized and become dynamically cold, so this scenario may better explain the observed kinematics.

Here we show that the observed stellar disk properties arise naturally by the partial capture of an extended molecular cloud that temporarily engulfs $\mathrm{Sgr}$ A* on a passage through the Galactic center rather than passing to one side of it. Cloud material passing on opposite sides of Sgr A* have oppositely-directed angular momenta, and gravitationally-induced collision of material downstream of Sgr A* reduces its angular momentum, permitting the captured material to settle into a compact configuration. The disks that form from this process are typically highly gravitational unstable, and so star formation can be expected to occur before the gas becomes dynamically cold. We also apply this formation scenario to the circumnuclear ring of gas which encircles Sgr A* with a rotational velocity of $\approx 100 \mathrm{~km} \mathrm{~s}^{-1}$ on a scale of 2-5 parsecs, and argue that it is just settling down after a recent capture event and is on the verge of forming stars.

\section{Cloud Capture by Sgr A*}

First we show that it is almost impossible for a cloud to be captured by Sgr $A^{*}$ and circularize to form the progenitor of the observed stellar disk without engulfing Sgr A* during the encounter. Consider an incoming cloud with velocity $v=100 v_{100} \mathrm{~km} \mathrm{~s}^{-1}$ and impact parameter $b$ at infinity, and assume that the cloud passes entirely to one side of Sgr $A^{*}$ as it begins the process of circularization. Tidal stretching and shocking convert the cloud's bulk kinetic energy to heat, but there is no mechanism able to reduce the mean angular momentum per unit mass $\sim b v$. Therefore the radius of the resulting disk $r_{d}$ satisfies

$$
r_{d}\left(\frac{G M}{r_{d}}\right)^{1 / 2} \approx b v
$$

where $M=4 \times 10^{6} \mathrm{M}_{\odot}$ is the mass of $\operatorname{Sgr} \mathrm{A}^{*}$. The observed stellar disk size, $r_{d} \approx 0.3 \mathrm{pc}$, then implies that the impact parameter $b \lesssim 0.7 v_{100}^{-1} \mathrm{pc}$. To avoid engulfing Sgr $\mathrm{A}^{*}$ during the capture, the cloud's radius must be much less than $b$, implying an initial density $n_{\mathrm{H}} \gtrsim$ $10^{7} \mathrm{~cm}^{-3}$ if the resultant disk is to have the mass $\sim 10^{5} \mathrm{M}_{\odot}$ inferred for the progenitor of the observed stellar disk. This scenario therefore requires a compact $(\lesssim 0.5 \mathrm{pc})$ and dense

$\left(\gtrsim 10^{7} \mathrm{~cm}^{-3}\right)$ interstellar cloud to be on a trajectory with impact parameter $\lesssim 1 \mathrm{pc}$ of Sgr $A^{*}$ - a unique event. 
Now consider the partial capture of clouds that engulf Sgr $A^{*}$ during their passage through the inner few parsecs of the Galaxy. The capture is enhanced by the gravitational focussing of material passing by $\mathrm{Sgr} \mathrm{A}^{*}$ and the subsequent collision of the gas just beyond Sgr A*, in a manner analogous to Bondi-Hoyle-Lyttleton accretion (Bondi \& Hoyle 1944). Fluid elements passing on opposite sides of Sgr $\mathrm{A}^{*}$ have oppositely-directed orbital angular momenta, so that the collision between them reduces their specific angular momentum. The efficiency of angular momentum cancellation depends on the density and velocity inhomogeneities in the incoming material. Velocity fluctuations are negligible because the velocity dispersion within molecular clouds is small compared to the highly supersonic bulk motion as clouds approach Sgr A*. Density inhomogneities in moleular clouds are large; however their effect is mitigated because the collision-induced accretion rate depends quadratically (rather than linearly) on the departure from homogeneity (Davies \& Pringle 1980). Numerical simulations have confirmed that the cancellation in the face of asymmetries of order unity is surprisingly efficient (Edgar 2004, and references therein). One key difference from classic Bondi-Hoyle-Lyttleton accretion flow is that the incoming gas is finite in extent. This means that tidal stretching of the incoming cloud may markedly change the outcome and that the flow does not develop long-term average behaviour.

Simulations are needed to address the details of the circularization process. For now we characterise the uncertain capture dynamics using two parameters: $\kappa$, the ratio of the captured mass to the Hoyle-Lyttleton estimate (Hoyle \& Lyttelton 1939), and $\lambda$, the fraction of the initial specific angular momentum retained by the captured material. These key parameters are sufficient to estimate the gross features of the resulting disk of captured material: its mass and size. While in principle $\kappa$ and $\lambda$ lie between 0 and $\sim 1$, our expectation is that the relative ease with which the gas can dissipate its bulk kinetic energy implies that $\kappa \sim 1$, wheras cancellation of angular momentum will be imperfect because of the inhomogeneities and finite extent of the incoming material, so that (perhaps) $\lambda \sim 0.3$.

Suppose then that an extended cloud with surface mass density $\Sigma_{\mathrm{cl}}$ equivalent to a column density of hydrogen nuclei $N_{24} \times 10^{24} \mathrm{H} \mathrm{cm}^{-2}$ is passing through the Galactic center with speed $v$. Cloud material with impact parameters less than about

$$
b_{0}=\frac{2 G M}{v^{2}}=3.4 v_{100}^{-2} \mathrm{pc},
$$

from Sgr $\mathrm{A}^{*}$ is captured, circularizes, and forms a disk of mass

$$
M_{d}=\pi \kappa b_{0}^{2} \Sigma_{\mathrm{cl}}=4.2 \times 10^{5} \kappa N_{24} v_{100}^{-4} \mathrm{M}_{\odot} .
$$

The outer radius of the disk, $r_{d}$, has specific angular momentum $r_{d} \sqrt{G M / r_{d}}$; the disk material at this radius corresponds to the matter with the largest angular momentum prior 
to capture, with impact parameter $\sim b_{0}$. The specific angular momentum of this material after circularization is $\lambda b_{0} v$, so the outer radius of the disk is

$$
r_{d}=2 \lambda^{2} b_{0}=6.9 \lambda^{2} v_{100}^{-2} \mathrm{pc}
$$

and the disk surface density is

$$
\Sigma_{d}=\frac{M_{d}}{\pi r_{d}^{2}}=\kappa \lambda^{-4} \Sigma_{\mathrm{cl}}=0.59 \kappa \lambda^{-4} \mathrm{~g} \mathrm{~cm}^{-2},
$$

Then Toomre's $Q$ is

$$
Q=\frac{c_{s} \Omega}{\pi G \Sigma_{d}}=0.11 T_{100}^{1 / 2} \frac{\lambda v_{100}^{3}}{\kappa N_{24}}
$$

with gravitational instability possible when $Q<1$. Here we have scaled the expression to a gas temperature $100 \mathrm{~K}$, a reasonable lower limit given the intense heating by the hot stars in the inner few parsecs of the Galactic center.

Eqs (3) -(6) show that the gross properties of the resultant disk depend on only two independent combinations of the four parameters $v_{100}, N_{24}, \kappa$ and $\lambda$, namely $v_{100} / \lambda$ and $\kappa N_{24} / v_{100}^{4}$. Note in particular that the temperature of the cloud does not affect the properties of the disk because the incident cloud material is highly supersonic.

In Fig. 2 we plot lines of constant disk mass, disk radius and Q in this two-dimensional parameter space, and indicate the regions corresponding to the stellar disk around Sgr A* and the circumnuclear ring (the latter is discussed in the next section).

The size of the stellar disk, $0.3 \mathrm{pc}$, implies that $v_{100} / \lambda \sim 5$. The inferred stellar mass, $1.4 \times 10^{4} \mathrm{M}_{\odot}$ (Paumard et al. 2006), places a lower limit on the disk mass: the disruptive effect of stellar winds and radiation from the first massive stars to form after the capture event, as well as potential losses of stars by scattering events suggest that the initial disk mass would likely have been at least several times higher, although SPH simulations indicate that the process of star formation may instead be nearly 100\% efficient (Nayakshin, Cuadra \& Springel 2007). Thus we consider progenitor disk masses in the range $10^{4}-10^{5} \mathrm{M}_{\odot}$, corresponding to $\kappa N_{24} / v_{100}^{4} \sim 2-20$. As noted earlier, the theoretical value of $\lambda$ is uncertain, but likely incident cloud speeds are between 50 and $100 \mathrm{~km} \mathrm{~s}^{-1}$, so the observed disk size implies that $\lambda \sim 0.1-$ 0.2 . Then the range of initial disk masses requires cloud column densities in the range $(2-60) \times 10^{24} \mathrm{~cm}^{-2}$. This is consistent with the observed range of column densities of the clouds currently in the Galactic center $\sim 10^{24}-10^{25} \mathrm{~cm}^{-2}$. 


\section{Discussion}

The scenario that we have outlined leads naturally to the formation of gravitationallyunstable rings of gas with the correct size and mass to explain the observed stellar disk. The mass estimate is robust as long as the capture radius $b_{0}$ is smaller than the size scale of the cloud, depending only on the idea that material that suffers significant deflections in the central potential will collide, shock and radiate away sufficient kinetic energy to become bound. Indeed, shocked, dense molecular gas cools efficiently for the $\lesssim 100 \mathrm{~km} \mathrm{~s}^{-1}$ shock speeds expected during circularization of the captured gas (e.g. Draine, Roberge \& Dalgarno 1983; Hollenbach \& McKee 1989), and within a few hundred years at most the temperature drops to $\sim 100 \mathrm{~K}$. This is shorter than the dynamical time scale $\sim 240(r / 0.1 \mathrm{pc})^{3 / 2} \mathrm{yr}$. This yields captured masses $\sim 10^{5} \mathrm{M}_{\odot}$ for cloud column densities $N_{H} \sim 10^{24} \mathrm{~cm}^{-2}$ and speeds $\sim 100 \mathrm{~km} \mathrm{~s}^{-1}$. The size of the resultant disk is set by the maximum angular momentum of the captured material after circularization (represented by the parameter $\lambda$ ). Although the values of $\lambda \sim 0.2$ needed to match the size of the Sgr A* stellar disks are reasonable, fluid-dynamical simulations are necessary to confirm this. The estimated $Q$ value of the pre-stellar disk is in the range 0.1-1, implying that the disk is gravitationally unstable and should fragment once the "turbulent" velocity dispersion of the gas settles down to the point that the effective $\mathrm{Q}$ (with $\Delta v$ substituted for the sound speed) becomes of order unity. As the cooling time is comparable to the dynamical time, stars are formed as the disk is circularizing, with a corresponding range of eccentricities and inclinations of the orbits (cf. Mapelli et al. 2008). This mechanism may explain why the observed stellar disk(s) are more disordered than would be produced by star formation in an intially kinematically cold disk. Subsequent orbit evolution due to gravitational interactions between stars should be minimal given that the stellar ages $\sim 6 \mathrm{Myr}$ (Paumard et al. 2006) are only a few hundred orbital periods (cf. Cuadra et al. 2008), and the resonant relaxation timescale exceeds $30 \mathrm{Myr}$ (Gürkan \& Hopman 2007).

Turning now to the circumnuclear ring, the observed CO (Harris et al. 1995) and HCN (Güsten et al. 1987) emission indicates an outer radius of $\sim 10 \mathrm{pc}$ and a total mass of $\sim 10^{5} \mathrm{M}_{\odot}$. More recent $\mathrm{HCN}$ observations imply that there is high density material close to the inner edge (Jackson et al. 1993; Christopher et al. 2005) suggesting that the mass may be closer to $10^{6} \mathrm{M}_{\odot}$. From Fig. 2 we infer $v_{100} / \lambda \approx 1$ and $\kappa N_{24} / v_{100}^{4} \approx 0.3-3$. The extent of the ring suggests an initial cloud speed towards the lower end of the $50-100 \mathrm{~km} \mathrm{~s}^{-1}$ range - otherwise $\lambda \sim 1$, and it would not be bound to Sgr A*. If we adopt $v \approx 50 \mathrm{~km} \mathrm{~s}^{-1}$, this implies $\lambda \sim 0.5$. This is reasonable given that at $50 \mathrm{~km} \mathrm{~s}^{-1}$ the Hoyle-Lyttleton radius is about 12 pc (see eq [2]), not much less than the probable cloud size. On this scale, one expects considerable asymmetry in the cloud material passing by Sgr A* during the capture event, with a corresponding reduction in the net cancellation of angular momentum during 
the capture process (cf. Bottema \& Sanders 1986; Sanders 1998). The disk mass requires column densities in the range $(50-500) \times 10^{24} \mathrm{~cm}^{-2}$, an order of magnitude higher than typical clouds in the Galactic center region. Note however that we ignored the gravitational effects of the evolved stellar cluster which become important beyond 2 pc (Genzel et al. 2000). This tends to increase the capture radius and captured mass, requiring larger incident cloud velocities, smaller $\lambda$ and smaller cloud column densities. At first sight our model suggests that the circumnuclear ring should be severely unstable to gravitational fragmentation, but this assumes that it has kinematically relaxed. The velocity dispersion of the ring is $\sim 30 \mathrm{~km} \mathrm{~s}^{-1}$, so that it is not unstable unless its mass is $\gtrsim 10^{6} \mathrm{M}_{\odot}$. There is little obvious sign of star formation, although methanol and water masers - signatures of the early phases of massive star formation - have recently been detected (Yusef-Zadeh et al. 2008). It appears that the circumnuclear ring is still in the process of settling down soon after formation. The ring's orbital time scale at $2 \mathrm{pc}$ is $\sim 10^{5} \mathrm{yr}$, so this implies that the age of the ring is $\lesssim 10^{6} \mathrm{yr}$. If this is so, the remains of the original interloper cloud should lie within $\sim 100 \mathrm{pc}$ of Sgr $\mathrm{A}^{*}$. One candidate is the $+50 \mathrm{~km} \mathrm{~s}^{-1}$ molecular cloud which extends along the plane from the Galactic center to $l \approx 0.2^{0}$ and consists of a number of bound cloudlets with a total mass of $\sim 10^{6} \mathrm{M}_{\odot}$ (Armstrong and Barrett 1985). This cloud is thought to lie about $30 \mathrm{pc}$ behind Sgr $A^{*}$, consistent with an interaction $\sim 3 \times 10^{5}$ years ago.

The age of the stellar disk, $\sim 5 \times 10^{6} \mathrm{yr}$ (Paumard et al. 2006), and the relative youth of the circumnuclear ring imply that the rate of encounters of massive clouds with Sgr A* is $\sim 10^{-6} \mathrm{yr}^{-1}$. This may have been been ongoing for a significant fraction fo the Galaxy's lifetime as the stellar population in the central parsec is consistent with roughly constant star formation over the past $12 \mathrm{Gyr}$ (Maness et al. 2007; but see also Blum et al. 2003), The inner $200 \mathrm{pc}$ of the Galaxy is rich in dense molecular clouds, many of which are on eccentric orbits (Bally et al. 1988; Oka et al. 1998; Martin et al. 2004). In addition to the $+50 \mathrm{~km} \mathrm{~s}^{-1}$ molecular cloud, the well-known 40,20 and $-30 \mathrm{~km} \mathrm{~s}^{-1}$ molecular clouds are all members of a disk population of molecular clouds distributed within the inner $30 \mathrm{pc}$ of Galactic center. Their non-circular, elongated motion is thought to be induced by the Galaxy's barred potential (e.g., Binney et al. 1991; Morris \& Serabyn 1996, and references therein), with dynamical friction aiding migration to the central regions of the Galaxy (Stark et al. 1991). Here star formation may instead occur through collisions between clouds, which create a high pressure environment suitable for cluster star formation (Tan \& McKee 2002). For example, the intense star formation apparent in Sgr B2 may have been triggered by the collision between the 65 and $80 \mathrm{~km} \mathrm{~s}^{-1}$ molecular clouds (Mehringer et al. 1993; Hasagawa et al. 1994), and the large proper motion of the Arches cluster may reflect this formation mechanism (Stolte et al. 2007). Apart from contributing to the central cusp in stellar density (Serabyn \& Morris 1996), the estimated infall rate, $\sim 0.4 \mathrm{M}_{\odot} \mathrm{yr}^{-1}$, is more than enough to 
bring $\mathrm{a} \sim 10^{5}-10^{6} \mathrm{M}_{\odot}$ cloud into the inner few parsec every few million years, where interaction with $\mathrm{Sgr} \mathrm{A}^{*}$ may produce a burst of star formation in a sub-parsec scale stellar disk.

\section{REFERENCES}

Armstrong, J. T. \& Barrett, A. H. 1985, ApJS, 57, 535

Bally, J., Stark, A. A., Wilson, R. W. \& Henkel, C. 1988, ApJ, 324, 223

Binney, J., Gerhard, O. E., Stark, A. A., Bally, J. \& Uchida, K. I. 1991, MNRAS, 252, 210

Blum, R. D., Ramirez, S. V., Sellgren, K. \& Olsen, K. 2003, ApJ, 597, 323

Bondi, H. \& Hoyle, F. 1944, MNRAS, 104, 273

Bottema, R. \& Sanders, R. H. 1986, A\&A, 158, 297

Christopher, M. H., Scoville, N. Z., Stolovy, S. R. \& Yun, M. S. 2005, ApJ, 622, 346

Cuadra, J., Armitage, P. J. \& Alexander, R. D. 2008, MNRAS, submitted (arXiv:0804.3596)

Cuadra, J., Nayakshin, S. \& Martins, F. 2008, MNRAS, 383, 458

Cuadra, J., Nayakshin, S., Springel, V. \& di Matteo, T. 2006, MNRAS, 366, 358

Davies, R. E. \& Pringle, J. E. 1980, MNRAS, 191, 599

Draine, B. T., Roberge, W. G. \& Dalgarno, A. 1983, ApJ, 264, 485

Edgar, R. 2004, New Astronomy Review, 48, 843

Fryxell, B. A. \& Taam, R. E. 1988, ApJ, 335, 862

Fujii, M., Iwasawa, M., Funato, Y. \& Makino, J. 2007, ApJ submitted (arXiv:0708.3719)

Gerhard, O. 2001, ApJ, 546, L39

Genzel, R., Pichon, C., Eckart, A., Gerhard, O. E. \& Ott, T. 2000, MNRAS, 317, 348

Genzel, R., Schdel, R., Ott, T., Eisenhauer, F., Hofmann, R., Lehnert, M., Eckart, A., Alexander, T., Sternberg, A., Lenzen, R., Clnet, Y., Lacombe, F., Rouan, D., Renzini, A. \& Tacconi-Garman, L. E. 2003, ApJ, 594, 812 
Gürkan, M. A. \& Hopman, C. 2007, MNRAS, 379, 1083

Gürkan, M. A. \& Rasio, F. A. 2005, ApJ, 628, 236

Harris, A. I., Jaffe, D. T., Silber, M. \& Genzel, R. 1985, ApJ, 294, L93

Hasegawa, T., Sato, F., Whiteoak, J. B. \& Miyawaki, R. 1994, ApJ, 429, L77

Hollenbach, D. J. \& McKee, C. F. 1989, ApJ, 342, 306

Hoyle, F. \& Lyttleton, R. A. 1939, Proc Camb Phil Soc, 34, 405

Jackson, J. M., Geis, N., Genzel, R., Harris, A. I., Madden, S., Poglitsch, A., Stacey, G. J. \& Townes, C. H. 1993, ApJ, 402, 173

Levin, Y. \& Beloborodov, A. M. 2003, ApJ, 590, L33

Mehringer, D. M., Palmer, P., Goss, W. M. \& Yusef-Zadeh, F. 1993, ApJ, 412, 684

Kim, S. S., Figer, D. F. \& Morris, M. 2004, ApJ, 607, L123

Krabbe, A., Genzel, R., Drapatz, S. \& Rotaciuc, V. 1991, ApJ, 382, L19

Krabbe, A., Genzel, R., Eckart, A., Najarro, F., Lutz, D., Cameron, M., Kroker, H., TacconiGarman, L. E., Thatte, N., Weitzel, L., Drapatz, S., Geballe, T., Sternberg, A. \& Kudritzki, R. 1995, ApJ, 447, L95

Lu, J. R., Ghez, A. M., Hornstein, S. D., Morris, M., Matthews, K., Thompson, D. J. \& Becklin, E. E. 2006, JPhCS, 54, 279

Maness, H., Martins, F., Trippe, S., Genzel, R., Graham, J. R., Sheehy, C., Salaris, M., Gillessen, S., Alexander, T., Paumard, T., Ott, T., Abuter, R. \& Eisenhauer, F. 2007, ApJ, 669, 1024

Mapelli, M., Hayfield, T., Mayer, L. \& Wadsley, J. 2008, MNRAS, submitted (arXiv:0805.0185)

Martin, C. L., Walsh, W. M., Xiao, K., Lane, A. P., Walker, C. K. \& Stark, A. A. 2004, ApJS, 150, 239

McMillan, S. L. W. \& Portegies Zwart, S. F. 2003, ApJ, 596, 314

Morris, M. 1993, ApJ, 408, 496

Morris, M., \& Serabyn, E. 1996, ARA\&A, 34, 645 
Mŭzić, K., Schödel, R., Eckart, A., Meyer, L. \& Zensus, A. 2008, A\&A, 482, 173

Nayakshin, S., Cuadra, J. \& Springel, V. 2007, MNRAS, 379, 21

Nayakshin, S., Dehnen, W., Cuadra, J. \& Genzel, R. 2006, MNRAS, 366, 1410

Nayakshin, S. \& Sunyaev, R. 2005, MNRAS, 364, L23

Oka, T., Hasegawa, T., Sato, F., Tsuboi, M. \& Miyazaki, A. 1998, ApJS, 118, 455

Paumard, T., Genzel, R., Martins, F., Nayakshin, S., Beloborodov, A. M., Levin, Y., Trippe, S., Eisenhauer, F., Ott, T., Gillessen, S., Abuter, R., Cuadra, J., Alexander, T. \& Sternberg, A. 2006, ApJ, 643, 1011

Portegies Zwart, S. F., McMillan, S. L. W. \& Gerhard, O. 2003, ApJ, 593, 352

Reid, M. J. \& Brunthaler, A. 2004, ApJ, 616, 872

Sanders, R. H. 1998, MNRAS, 294, 35

Schödel, R., Ott, T., Genzel, R., Eckart, A., Mouawad, N. \& Alexander, T. 2003, ApJ, 596, 1015

Serabyn, E. \& Morris, M. 1996, Nature, 382, 602

Stark, A. A., Bally, J., Gerhard, O. E. \& Binney, J. 1991, MNRAS, 248, 14P

Stolte, A., Ghez, A. M., Morris, M., Lu, J. R., Brandner, W. \& Matthews, K. 2008, ApJ, 675,1278

Taam, R. E. \& Fryxell, B. A. 1989, ApJ, 339, 297

Tan, J. C. \& McKee, C. F. 2004, in The Formation and Evolution of Massive Young Star Clusters, ed. H. J. G. L. M. Lamers, L. J. Smith \& A. Nota (San Francisco: ASP), 263

Yusef-Zadeh, F., Braatz, J., Wardle, M., \& Roberts, D. 2008, ApJL submitted

Yusef-Zadeh, F. \& Wardle, M. 2008, in Massive Star Formation: Observations Confront Theory, ed. H. Beuther, H. Linz \& T. Henning (San Francisco: ASP), 361 

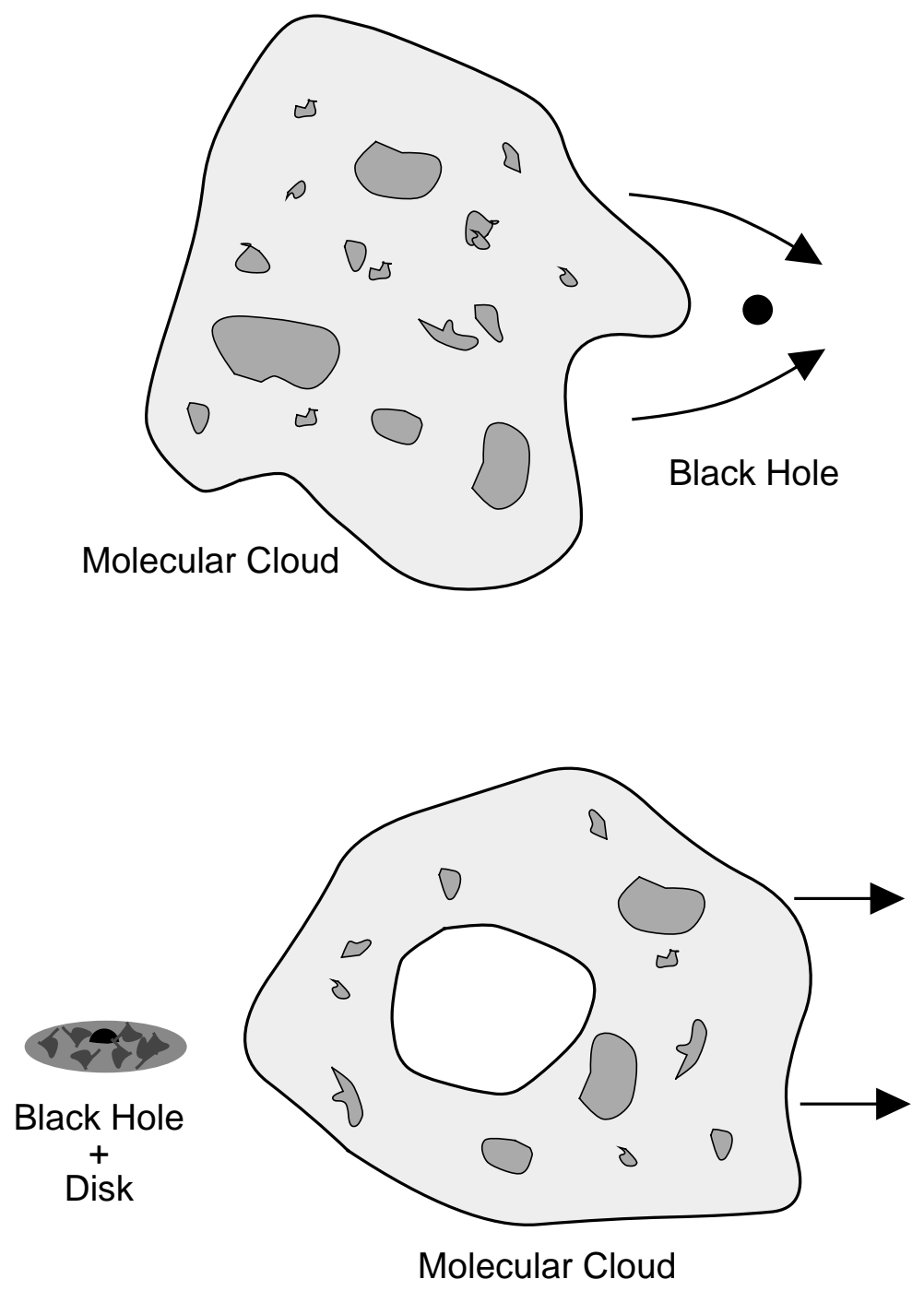

Fig. 1.- A schematic diagram of a cloud impacting Sgr A*. The upper panel indicates the gravitational focusing of incoming molecular cloud material (incident from the left). The lower panel shows the carved-out inner region of the cloud that has been captured by Sgr $\mathrm{A}^{*}$ and circularized to form a disk. The outer region of the cloud continues its motion in the direction away from Sgr A*. 


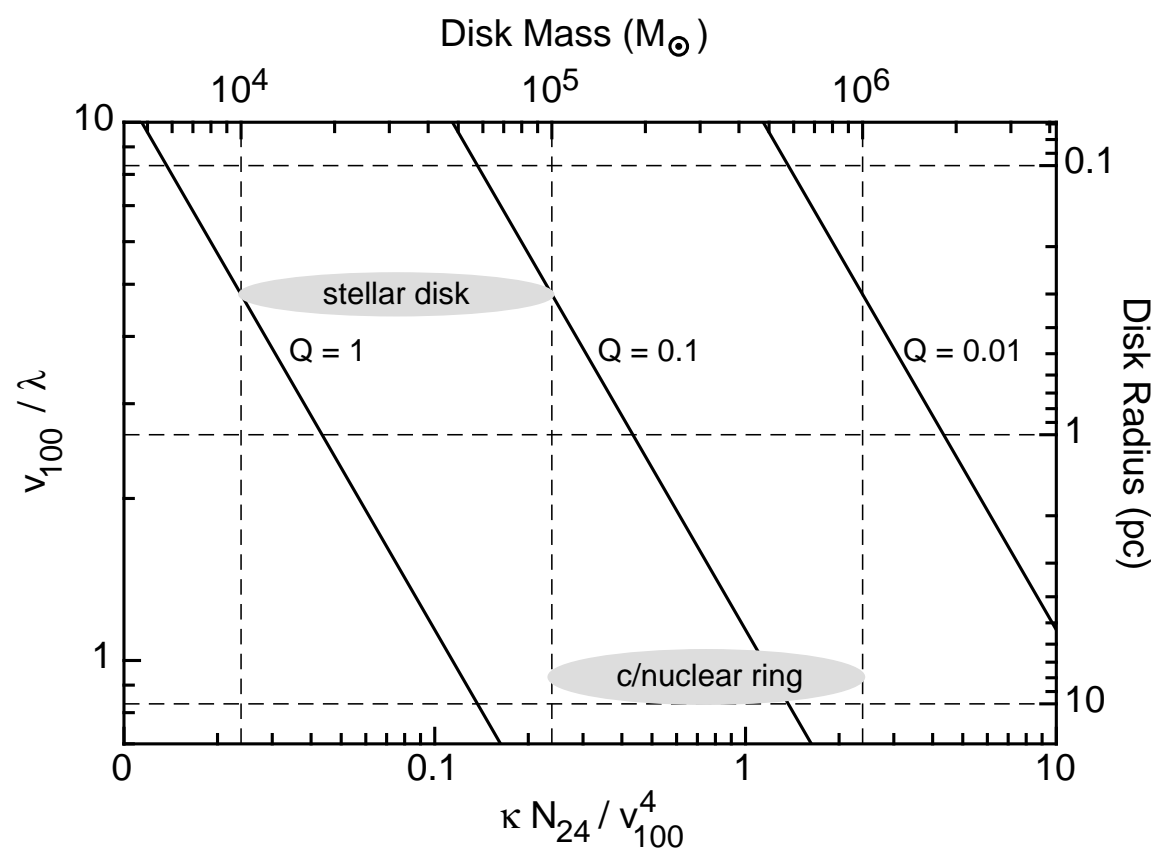

Fig. 2.- The mass and radius of disks formed by the partial capture of interloper clouds that temporarily engulf Sgr A* are determined by the cloud speed $v=v_{100} \times 100 \mathrm{~km} \mathrm{~s}^{-1}$ and column density $N_{\mathrm{H}}=N_{24} \times 10^{24} \mathrm{~cm}^{-2} . \kappa$ is the fraction of the cloud material with impact parameters less than $2 G M / v^{2}$ that is captured, and $\lambda$ is the fraction of angular momentum remaining after circularization of the captured material. Horizontal and vertical dashes indicate lines of constant disk radius and mass, respectively. Diagonal lines are labelled by their value of $Q=c_{s} \Omega / \pi G \Sigma_{d}$ assuming a temperature of $100 \mathrm{~K}$ (see text); the region to the right of the $\mathrm{Q}=1$ line are unstable to gravitational fragmentation. Grey shaded regions indicate the disk parameters for the the stellar disk close to Sgr A* and for the circumnuclear ring. 